\title{
ON VARIANCES OF PARTIAL VOLUMES OF THE TYPICAL CELL OF A POISSON-VORONOI TESSELLATION AND LARGE-DIMENSIONAL VOLUME DEGENERACY
}

\author{
YI-CHING YAO, * Academia Sinica and National Chengchi University
}

\begin{abstract}
For a typical cell of a homogeneous Poisson-Voronoi tessellation in $\mathbb{R}^{d}$, it is shown that the variance of the volume of the intersection of the typical cell with any measurable subset of $\mathbb{R}^{d}$ is bounded by the variance of the volume of the typical cell. It is also shown that the variance of the volume of the intersection of the typical cell with a translation of itself is bounded by four times the variance of the volume of the typical cell. These bounds are applied to show large-dimensional volume degeneracy as $d$ tends to $\infty$. An extension to the $k$ th nearest-point Poisson-Voronoi tessellation for $k \geq 2$ is also considered.
\end{abstract}

Keywords: Poisson process; Voronoi tessellation; typical cell; geometric covariogram; nearest neighbor

2010 Mathematics Subject Classification: Primary 60D05

Secondary 60G55

\section{Introduction}

Associated with a homogeneous Poisson point process $\Pi=\Pi_{d}$ in $\mathbb{R}^{d}$ with intensity $\lambda>0$, the Voronoi tessellation is defined as $\left\{C_{X}(\Pi): X \in \Pi\right\}$, where $C_{X}(\Pi)$, the Voronoi cell of $\Pi$ centered at $X$, is given by $C_{X}(\Pi)=\left\{x \in \mathbb{R}^{d}:\|x-X\| \leq\left\|x-X^{\prime}\right\|\right.$ for all $\left.X^{\prime} \in \Pi\right\}$, $\|\cdot\|=\|\cdot\|_{d}$ being the $d$-dimensional Euclidean norm. A 'typical cell' refers to a uniform random member (translated so as to be centered at the origin) of the Voronoi tessellation, and can be explicitly described (cf. [5]) by $C_{t}=C_{t, d}:=C_{\mathbf{0}}(\Pi \cup\{\mathbf{0}\})=\left\{x \in \mathbb{R}^{d}:\|x\| \leq\|x-X\|\right.$ for all $X \in \Pi\}$, the Voronoi cell centered at the origin $\mathbf{0}$ after adding $\mathbf{0}$ to $\Pi$. A subscript $d$ is attached to $\Pi, C_{t},\|\cdot\|$, and $\mu$ (the Lebesgue measure on $\mathbb{R}^{d}$ ) in order to emphasize dependence on $d$, which is suppressed in the proofs for ease of notation when no danger of confusion arises.

In the literature on the moments and distributions of geometric characteristics of the typical cell most of the analytic and simulation studies have been concerned with the two important low-dimensional cases $d=2$ and $d=3$ (see [9] and [12] for a comprehensive review). At the other extreme, Newman et al. [11] first studied the large-dimensional limit of $\mu_{d}\left(C_{t, d}\right)$, the $d$-dimensional Lebesgue measure ( $d$-volume) of $C_{t, d}$, and showed that, as $d \rightarrow \infty, \mu_{d}\left(C_{t, d}\right)$ converges in distribution to $1 / \lambda$. (In fact, their Theorem 10 implies that, as $d \rightarrow \infty, \mu_{d}\left(C_{t, d}\right.$ ) converges in $L^{p}$ to $1 / \lambda$ for all $p>0$. Here a sequence of real-valued random variables $\left\{Y_{n}\right\}$, not necessarily defined on the same probability space, is said to converge in $L^{p}$ to a constant $\gamma$ as $n \rightarrow \infty$ if $\lim _{n \rightarrow \infty} \mathrm{E}\left[\left|Y_{n}-\gamma\right|^{p}\right]=0$.) Recently, Alishahi and Sharifitabar [1] established

Received 18 May 2009; revision received 8 December 2009.

* Postal address: Institute of Statistical Science, Academia Sinica, Taipei 115, Taiwan, R.O.C.

Email address: yao@stat.sinica.edu.tw 
the following sharp bound on the variance of $\mu_{d}\left(C_{t, d}\right)$ :

$$
\operatorname{var}\left(\mu_{d}\left(C_{t, d}\right)\right)=\mathrm{E}\left[\left(\mu_{d}\left(C_{t, d}\right)-\frac{1}{\lambda}\right)^{2}\right]<\frac{c}{\lambda^{2} \sqrt{d}}\left(\frac{4}{3 \sqrt{3}}\right)^{d} \text { for all } d,
$$

where $c$ is a constant. By [1, Remark 3.1], $c=5$ suffices for (1) to hold. Note that, to establish (1), they used the Blaschke-Petkantschin formula (see, e.g. [6, Chapter 4]) to obtain an integral formula for $\mathrm{E}\left[\left\{\mu_{d}\left(C_{t, d}\right)\right\}^{2}\right]$, while a similar integral representation was derived earlier by Gilbert [4, Formula (14)]. Alishahi and Sharifitabar further showed that, for each fixed $u>0$, as $d \rightarrow \infty$, the $d$-volume of the intersection of $C_{t, d}$ with a ball of volume $u$ centered at the origin converges in $L^{2}$ to $\lambda^{-1}\left(1-\mathrm{e}^{-\lambda u}\right)$. These results indicate the phenomenon of large-dimensional volume degeneracy of the typical cell.

The geometric covariogram of a bounded measurable set $K \subset \mathbb{R}^{d}$ is the function $g_{K}(v)=$ $\mu_{d}(K \cap(K+v)), v \in \mathbb{R}^{d}$, which was introduced by Matheron [8], who also asked the question of whether the geometric covariogram uniquely determines a convex set up to translations and reflections. While Bianchi [3] found counterexamples for $d \geq 4, g_{K}$ clearly contains much information about the geometric characteristics of $K$ and can uniquely determine $K$ for some classes of convex sets. See Averkov and Bianchi [2] and the references therein. Alishahi and Sharifitabar [1] considered the (random) geometric covariogram of the typical cell, $g_{C_{t, d}}(v)=\mu_{d}\left(C_{t, d} \cap\left(C_{t, d}+v\right)\right), v \in \mathbb{R}^{d}$, which is a random function. (Note that Lantuéjoul [7, p. 23] defined the geometric covariogram of a random set $K$ by taking the expectation $\mathrm{E}\left[\mu_{d}(K \cap(K+v))\right]$.) For $r>0$ and $v_{d} \in \mathbb{R}^{d}$ with $\left\|v_{d}\right\|_{d}=1$, they proved that $\lim _{d \rightarrow \infty} \mathrm{E}\left[g_{C_{t, d}}\left(r v_{d}\right)\right]=c(r, \lambda)$, where

$$
c(r, \lambda)=\frac{1}{\lambda \sqrt{2 \pi}} \int_{-\infty}^{\infty} \frac{\mathrm{d} t}{\exp \left(t^{2} / 2\right) \Phi(t)+\exp \left((t-r \sqrt{2 \pi \mathrm{e}})^{2} / 2\right)(1-\Phi(t-r \sqrt{2 \pi \mathrm{e}}))},
$$

$\Phi(\cdot)$ being the standard normal cumulative distribution function. An interesting question was raised in [1, p. 934] as to whether $g_{C_{t, d}}\left(v_{d}\right)$ becomes deterministic for large $d$. Indeed, a more general question is to determine those partial volumes of the typical cell which become deterministic for large $d$.

In the next section we show that $\mu_{d}\left(C_{t, d} \cap S_{1}\right)$ and $\mu_{d}\left(C_{t, d} \cap S_{2}\right)$ have a nonnegative covariance for any measurable subsets $S_{1}$ and $S_{2}$ of $\mathbb{R}^{d}$, implying that $\operatorname{var}\left(\mu_{d}\left(C_{t, d} \cap S_{1}\right)\right) \leq$ $\operatorname{var}\left(\mu_{d}\left(C_{t, d} \cap S_{2}\right)\right) \leq \operatorname{var}\left(\mu_{d}\left(C_{t, d}\right)\right)$ if $S_{1} \subset S_{2} \subset \mathbb{R}^{d}$. It then follows that, as $d \rightarrow \infty$, $\mu_{d}\left(C_{t, d} \cap A_{d}\right)-\mathrm{E}\left[\mu_{d}\left(C_{t, d} \cap A_{d}\right)\right]$ converges in $L^{2}$ to 0 for any sequence of measurable sets $A_{d} \subset \mathbb{R}^{d}, d=1,2, \ldots$ We further strengthen the $L^{2}$ convergence to $L^{p}$ convergence for all $p>0$. We also discuss conditions under which $\mu_{d}\left(C_{t, d} \cap A_{d}\right) / \mathrm{E}\left[\mu_{d}\left(C_{t, d} \cap A_{d}\right)\right]$ converges in $L^{2}$ to 1 as $d \rightarrow \infty$.

In Section 3 we consider the geometric covariogram of the typical cell and show that $\operatorname{var}\left(g_{C_{t, d}}(v)\right) \leq 4 \operatorname{var}\left(\mu_{d}\left(C_{t, d}\right)\right)$ for all $v \in \mathbb{R}^{d}$. Together with (1), this inequality implies that, as $d \rightarrow \infty, g_{C_{t, d}}\left(v_{d}\right)-\mathrm{E}\left[g_{C_{t, d}}\left(v_{d}\right)\right]$ converges in $L^{2}$ to 0 for any sequence of $v_{d} \in \mathbb{R}^{d}$, $d=1,2, \ldots$, which answers in the affirmative the aforementioned question raised in [1, p. 934]. (The $L^{2}$ convergence can in fact be strengthened to $L^{p}$ convergence for all $p>0$.)

In Section 4 we consider the typical cell (centered at the origin) of the $k$ th nearest-point Poisson-Voronoi tessellation, $k=1,2, \ldots$, defined by

$$
C_{t, d}^{(k)}:=\left\{x \in \mathbb{R}^{d}: \#\{X \in \Pi:\|x-X\|<\|x\|\}=k-1\right\} .
$$

(See [12, Section 3.3.2] for a brief discussion.) Note that $C_{t, d}^{(1)}=C_{t, d}$. We show that, for $k=1,2, \ldots$ and $p>0, \mu_{d}\left(C_{t, d}^{(k)}\right)$ converges in $L^{p}$ to $1 / \lambda$ as $d \rightarrow \infty$, from which follows a 
large-dimensional independent and identically distributed property for nearest-neighbor counts in two-ensemble Poisson point processes (see Remark 11).

\section{Nonnegative covariance of partial volumes of the typical cell and its implications}

Theorem 1. For any measurable subsets $S_{1}$ and $S_{2}$ of $\mathbb{R}^{d}$, we have

$$
\operatorname{cov}\left(\mu_{d}\left(C_{t, d} \cap S_{1}\right), \mu_{d}\left(C_{t, d} \cap S_{2}\right)\right) \geq 0 .
$$

If $S_{1} \subset S_{2} \subset \mathbb{R}^{d}$ then

$$
\operatorname{var}\left(\mu_{d}\left(C_{t, d} \cap S_{1}\right)\right) \leq \operatorname{var}\left(\mu_{d}\left(C_{t, d} \cap S_{2}\right)\right) \leq \operatorname{var}\left(\mu_{d}\left(C_{t, d}\right)\right) .
$$

Proof. Without loss of generality, assume that $\lambda=1$. For ease of notation, the subscript $d$ in $C_{t, d}, \mu_{d}$, and $\|\cdot\|_{d}$ is suppressed. Letting $\mathbf{1}_{S}$ denote the indicator function of the set $S$ and $B_{v}$ denote the ball centered at $v$ with radius $\|v\|$ (i.e. $B_{v}=\left\{x \in R^{d}:\|x-v\|<\|v\|\right\}$ ), we have, for $x, y \in \mathbb{R}^{d}$,

$$
\begin{aligned}
\mathrm{E}\left[\mathbf{1}_{C_{t}}(x)-\mathrm{e}^{-\mu\left(B_{x}\right)}\right]\left[\mathbf{1}_{C_{t}}(y)-\mathrm{e}^{-\mu\left(B_{y}\right)}\right] & =\mathrm{E}\left[\mathbf{1}_{C_{t}}(x) \mathbf{1}_{C_{t}}(y)\right]-\mathrm{e}^{-\mu\left(B_{x}\right)-\mu\left(B_{y}\right)} \\
& =\mathrm{e}^{-\mu\left(B_{x} \cup B_{y}\right)}-\mathrm{e}^{-\mu\left(B_{x}\right)-\mu\left(B_{y}\right)} \\
& \geq 0 .
\end{aligned}
$$

Since, for any measurable set $S \subset \mathbb{R}^{d}$,

$$
\mu\left(C_{t} \cap S\right)-\mathrm{E}\left[\mu\left(C_{t} \cap S\right)\right]=\int_{S}\left[\mathbf{1}_{C_{t}}(x)-\mathrm{e}^{-\mu\left(B_{x}\right)}\right] \mathrm{d} x,
$$

it follows by Fubini's theorem and (4) that

$$
\begin{aligned}
\operatorname{cov}\left(\mu\left(C_{t} \cap S_{1}\right), \mu\left(C_{t} \cap S_{2}\right)\right) & =\mathrm{E} \int_{S_{1}}\left[\mathbf{1}_{C_{t}}(x)-\mathrm{e}^{-\mu\left(B_{x}\right)}\right] \mathrm{d} x \int_{S_{2}}\left[\mathbf{1}_{C_{t}}(y)-\mathrm{e}^{-\mu\left(B_{y}\right)}\right] \mathrm{d} y \\
& =\int_{S_{1} \times S_{2}} \mathrm{E}\left[\mathbf{1}_{C_{t}}(x)-\mathrm{e}^{-\mu\left(B_{x}\right)}\right]\left[\mathbf{1}_{C_{t}}(y)-\mathrm{e}^{-\mu\left(B_{y}\right)}\right] \mathrm{d} x \mathrm{~d} y \\
& \geq 0 .
\end{aligned}
$$

If $S_{1} \subset S_{2}$ then $\mu\left(C_{t} \cap S_{2}\right)=\mu\left(C_{t} \cap S_{1}\right)+\mu\left(C_{t} \cap\left(S_{2} \backslash S_{1}\right)\right)$, so that

$$
\begin{aligned}
& \operatorname{var}\left(\mu\left(C_{t} \cap S_{2}\right)\right) \\
& \quad=\operatorname{var}\left(\mu\left(C_{t} \cap S_{1}\right)\right)+\operatorname{var}\left(\mu\left(C_{t} \cap\left(S_{2} \backslash S_{1}\right)\right)\right)+2 \operatorname{cov}\left(\mu\left(C_{t} \cap S_{1}\right), \mu\left(C_{t} \cap\left(S_{2} \backslash S_{1}\right)\right)\right) \\
& \quad \geq \operatorname{var}\left(\mu\left(C_{t} \cap S_{1}\right)\right) .
\end{aligned}
$$

This completes the proof.

Remark 1. Note that the inequality in (4) is strict unless the origin $\mathbf{0}$ lies in the line segment connecting $x$ and $y$. It follows that, for measurable subsets $S_{1}$ and $S_{2}$ of $\mathbb{R}^{d}$ with $d \geq 2$, $\operatorname{cov}\left(\mu_{d}\left(C_{t, d} \cap S_{1}\right), \mu_{d}\left(C_{t, d} \cap S_{2}\right)\right)>0$ if $\mu_{d}\left(S_{1}\right)>0$ and $\mu_{d}\left(S_{2}\right)>0$. (For $d=1$, $\mu_{d}\left(C_{t, d} \cap S_{1}\right)$ and $\mu_{d}\left(C_{t, d} \cap S_{2}\right)$ are independent if $S_{1} \subset(-\infty, 0)$ and $S_{2} \subset(0, \infty)$.) 
Remark 2. By direct calculations, Alishahi and Sharifitabar [1] showed that, as $d \rightarrow \infty$, $\operatorname{var}\left(\mu_{d}\left(C_{t, d} \cap S\right)\right)$ decays to 0 at a geometric rate when $S$ is a ball centered at $\mathbf{0}$. By Theorem 1 , this is true for general $S$. While a small value of $\operatorname{var}\left(\mu_{d}\left(C_{t, d} \cap S\right)\right)$ implies that $\mu_{d}\left(C_{t, d} \cap S\right)$ is close to its mean with high probability, it does not say much about volume degeneracy when $\mathrm{E}\left[\mu_{d}\left(C_{t, d} \cap S\right)\right]$ is small, in which case a more relevant measure of volume degeneracy is the coefficient of variation

$$
\mathrm{CV}\left(\mu_{d}\left(C_{t, d} \cap S\right)\right):=\frac{\sqrt{\operatorname{var}\left(\mu_{d}\left(C_{t, d} \cap S\right)\right)}}{\mathrm{E}\left[\mu_{d}\left(C_{t, d} \cap S\right)\right]},
$$

since a small value of $\mathrm{CV}\left(\mu_{d}\left(C_{t, d} \cap S\right)\right)$ implies that $\mu_{d}\left(C_{t, d} \cap S\right) / \mathrm{E}\left[\mu_{d}\left(C_{t, d} \cap S\right)\right]$ is close to 1 with high probability. With $r_{d}=d^{-1 / 4}(4 / 3 \sqrt{3})^{d / 2}$, it follows from (1) that $\lim _{d \rightarrow \infty} \mathrm{CV}\left(\mu_{d}\left(C_{t, d} \cap A_{d}\right)\right)=0$ whenever measurable sets $A_{d} \subset \mathbb{R}^{d}$ satisfy

$$
\lim _{d \rightarrow \infty} \frac{\mathrm{E}\left[\mu_{d}\left(C_{t, d} \cap A_{d}\right)\right]}{r_{d}}=\infty
$$

It would be of interest to find the optimal rate of $r_{d}$ satisfying the above property.

Remark 3. As an example, consider $A_{d}=(0, \infty)^{n_{d}} \times \mathbb{R}^{d-n_{d}}=\left\{\left(x_{1}, \ldots, x_{d}\right) \in \mathbb{R}^{d}: x_{i}>0\right.$, $\left.i=1, \ldots, n_{d}\right\}$, where $1 \leq n_{d} \leq d$ is an integer. Note that the total volume of the typical cell $\mu_{d}\left(C_{t, d}\right)$ can be divided into $2^{n_{d}}$ parts, one of which is $\mu_{d}\left(C_{t, d} \cap A_{d}\right)$ and all of which have the same distribution by symmetry. It follows that

$$
\begin{gathered}
\lambda^{-1}=\mathrm{E}\left[\mu_{d}\left(C_{t, d}\right)\right]=2^{n_{d}} \mathrm{E}\left[\mu_{d}\left(C_{t, d} \cap A_{d}\right)\right], \\
\operatorname{var}\left(\mu_{d}\left(C_{t, d}\right)\right) \geq 2^{n_{d}} \operatorname{var}\left(\mu_{d}\left(C_{t, d} \cap A_{d}\right)\right),
\end{gathered}
$$

since the covariance of each pair of the $2^{n_{d}}$ parts is nonnegative. We have, by (1),

$$
\operatorname{var}\left(\mu_{d}\left(C_{t, d} \cap A_{d}\right)\right) \leq \frac{c}{\lambda^{2} \sqrt{d}} 2^{-n_{d}}\left(\frac{4}{3 \sqrt{3}}\right)^{d} .
$$

While the bound in (6) is crude, (5) and (6) imply that $\lim _{d \rightarrow \infty} \operatorname{CV}\left(\mu_{d}\left(C_{t, d} \cap A_{d}\right)\right)=0$ if $\limsup _{d \rightarrow \infty} n_{d} / d<\log 27 / \log 4-2$.

Remark 4. By [11, Theorem 10], $\lim _{d \rightarrow \infty} \mathrm{E}\left[\mu_{d}\left(C_{t, d}\right)\right]^{n}=\lambda^{-n}, n=1,2, \ldots$, which implies that $\mu_{d}\left(C_{t, d}\right)$ converges in $L^{p}$ to $\lambda^{-1}$ for all $p>0$. We now show that, for any sequence of measurable sets $A_{d} \subset \mathbb{R}^{d}, \mu_{d}\left(C_{t, d} \cap A_{d}\right)-\mathrm{E}\left[\mu_{d}\left(C_{t, d} \cap A_{d}\right)\right]$ converges in $L^{p}$ to 0 as $d \rightarrow \infty$ for all $p>0$. Letting $U_{d}:=\mu_{d}\left(C_{t, d} \cap A_{d}\right), m_{d}:=\mathrm{E}\left(U_{d}\right)$, and $V_{d}:=\mu_{d}\left(C_{t, d}\right)$, and noting that $m_{d} \leq \mathrm{E}\left(V_{d}\right)=1 / \lambda$, we have, for $p>2$,

$$
\begin{aligned}
& \mathrm{E}\left|U_{d}-m_{d}\right|^{p}=\mathrm{E}\left|U_{d}-m_{d}\right|^{p} \mathbf{1}_{\left\{\left|U_{d}-m_{d}\right| \leq 2 / \lambda\right\}}+\mathrm{E}\left|U_{d}-m_{d}\right|^{p} \mathbf{1}_{\left\{\left|U_{d}-m_{d}\right|>2 / \lambda\right\}}, \\
& \mathrm{E}\left|U_{d}-m_{d}\right|^{p} \mathbf{1}_{\left\{\left|U_{d}-m_{d}\right| \leq 2 / \lambda\right\}} \leq\left(\frac{2}{\lambda}\right)^{p-2} \mathrm{E}\left|U_{d}-m_{d}\right|^{2} \mathbf{1}_{\left\{\left|U_{d}-m_{d}\right| \leq 2 / \lambda\right\}} \\
& \leq\left(\frac{2}{\lambda}\right)^{p-2} \operatorname{var}\left(U_{d}\right) \\
& \rightarrow 0 \text { as } d \rightarrow \infty,
\end{aligned}
$$




$$
\begin{aligned}
\mathrm{E}\left|U_{d}-m_{d}\right|^{p} \mathbf{1}_{\left\{\left|U_{d}-m_{d}\right|>2 / \lambda\right\}} \leq & \mathrm{E}\left|U_{d}-m_{d}\right|^{p} \mathbf{1}_{\left\{U_{d}>2 / \lambda\right\}} \\
& \quad\left(\text { since }\left|U_{d}-m_{d}\right|>2 / \lambda \text { implies that } U_{d}>2 / \lambda\right) \\
& \leq \mathrm{E}\left|V_{d}-m_{d}\right|^{p} \mathbf{1}_{\left\{V_{d}>2 / \lambda\right\}} \quad\left(\text { since } V_{d} \geq U_{d}\right) \\
& \leq 2^{p} \mathrm{E}\left|V_{d}-\lambda^{-1}\right|^{p} \mathbf{1}_{\left\{V_{d}>2 / \lambda\right\}} \\
& \leq 2^{p} \mathrm{E}\left|V_{d}-\lambda^{-1}\right|^{p} \\
& \rightarrow 0 \quad \text { as } d \rightarrow \infty,
\end{aligned}
$$

where the second-to-last inequality follows from the fact that $V_{d}>2 / \lambda$ implies that $2\left(V_{d}-\right.$ $1 / \lambda)>V_{d}-m_{d}$. This proves that $\mu_{d}\left(C_{t, d} \cap A_{d}\right)-\mathrm{E}\left[\mu_{d}\left(C_{t, d} \cap A_{d}\right)\right]$ converges in $L^{p}$ to 0 as $d \rightarrow \infty$ for all $p>0$.

Remark 5. The following simple fact may have something to do with the volume degeneracy property of the typical cell. Let $Q_{d}$ denote a typical point of the Poisson point process $\Pi_{d}$, and let $X_{i, d} \in \Pi_{d}$ denote $Q_{d}$ 's $i$ th nearest neighbor. Then $X_{i, d}-Q_{d}, i=1,2, \ldots$, are asymptotically orthogonal as $d \rightarrow \infty$, i.e. for $i \neq j$,

$$
\frac{\left\langle X_{i, d}-Q_{d}, X_{j, d}-Q_{d}\right\rangle_{d}}{\left\|X_{i, d}-Q_{d}\right\|_{d}\left\|X_{j, d}-Q_{d}\right\|_{d}} \rightarrow 0 \quad \text { in probability as } d \rightarrow \infty,
$$

where $\langle\cdot, \cdot\rangle_{d}$ is the inner product associated with the norm $\|\cdot\|_{d}$. This fact is related to a result of Newman et al. [11] which states that the number of points among $X_{i, d}, i=1,2, \ldots$, that have $Q_{d}$ as their nearest neighbor has a limiting Poisson distribution with mean 1 as $d \rightarrow \infty$. Further extensions to general $k$ th nearest-neighbor counts with respect to the $l^{p}$ distance $(1 \leq p \leq \infty)$ can be found in [10] and [13], where $p=\infty$ refers to the supnorm distance.

\section{Bound on the variance of the geometric covariogram of the typical cell}

Theorem 2. For all $v \in \mathbb{R}^{d}$, we have

$$
\operatorname{var}\left(\mu_{d}\left(C_{t, d} \cap\left(C_{t, d}+v\right)\right)\right) \leq 4 \operatorname{var}\left(\mu_{d}\left(C_{t, d}\right)\right) .
$$

Proof. Without loss of generality, assume that $\lambda=1$. For ease of notation, we suppress the subscript $d$ in $C_{t, d}, \mu_{d}$, and $\|\cdot\|_{d}$. Recall that $B_{v}=\left\{x \in \mathbb{R}^{d}:\|x-v\|<\|v\|\right\}$. Since

$$
\mu\left(C_{t} \cap\left(C_{t}+v\right)\right)=\int_{\mathbb{R}^{d}} \mathbf{1}_{C_{t}}(x) \mathbf{1}_{C_{t}}(x-v) \mathrm{d} x
$$

and

$$
\mathrm{E}\left[\mu\left(C_{t} \cap\left(C_{t}+v\right)\right)\right]=\int_{\mathbb{R}^{d}} \mathrm{e}^{-\mu\left(B_{x} \cup B_{x-v}\right)} \mathrm{d} x,
$$

we have, by Fubini's theorem,

$$
\begin{aligned}
\operatorname{var} & \left(\mu\left(C_{t} \cap\left(C_{t}+v\right)\right)\right) \\
& =\mathrm{E} \int_{\mathbb{R}^{d}}\left[\mathbf{1}_{C_{t}}(x) \mathbf{1}_{C_{t}}(x-v)-\mathrm{e}^{-\mu\left(B_{x} \cup B_{x-v}\right)}\right] \mathrm{d} x \int_{\mathbb{R}^{d}}\left[\mathbf{1}_{C_{t}}(y) \mathbf{1}_{C_{t}}(y-v)-\mathrm{e}^{-\mu\left(B_{y} \cup B_{y-v}\right)}\right] \mathrm{d} y \\
& =\int_{\mathbb{R}^{d} \times \mathbb{R}^{d}}\left[\mathrm{E} \mathbf{1}_{C_{t}}(x) \mathbf{1}_{C_{t}}(x-v) \mathbf{1}_{C_{t}}(y) \mathbf{1}_{C_{t}}(y-v)-\mathrm{e}^{-\mu\left(B_{x} \cup B_{x-v}\right)-\mu\left(B_{y} \cup B_{y-v}\right)}\right] \mathrm{d} x \mathrm{~d} y \\
& =\int_{\mathbb{R}^{d} \times \mathbb{R}^{d}}\left[\mathrm{e}^{-\mu\left(B_{x} \cup B_{x-v} \cup B_{y} \cup B_{y-v}\right)}-\mathrm{e}^{-\mu\left(B_{x} \cup B_{x-v}\right)-\mu\left(B_{y} \cup B_{y-v}\right)}\right] \mathrm{d} x \mathrm{~d} y .
\end{aligned}
$$


Note that

$$
\begin{aligned}
\mu\left(B_{x}\right. & \left.\cup B_{x-v}\right)+\mu\left(B_{y} \cup B_{y-v}\right)-\mu\left(B_{x} \cup B_{x-v} \cup B_{y} \cup B_{y-v}\right) \\
& =\mu\left(\left(B_{x} \cup B_{x-v}\right) \cap\left(B_{y} \cup B_{y-v}\right)\right) \\
& =\mu\left(\left(B_{x} \cap B_{y}\right) \cup\left(B_{x} \cap B_{y-v}\right) \cup\left(B_{x-v} \cap B_{y}\right) \cup\left(B_{x-v} \cap B_{y-v}\right)\right) \\
& \leq \mu\left(B_{x} \cap B_{y}\right)+\mu\left(B_{x} \cap B_{y-v}\right)+\mu\left(B_{x-v} \cap B_{y}\right)+\mu\left(B_{x-v} \cap B_{y-v}\right),
\end{aligned}
$$

so that, by (7),

$$
\begin{aligned}
& \operatorname{var}\left(\mu\left(C_{t} \cap\left(C_{t}+v\right)\right)\right) \\
& =\int_{\mathbb{R}^{d} \times \mathbb{R}^{d}} \mathrm{e}^{-\mu\left(B_{x} \cup B_{x-v} \cup B_{y} \cup B_{y-v}\right)} \\
& \quad \times\left[1-\mathrm{e}^{-\mu\left(B_{x} \cup B_{x-v}\right)-\mu\left(B_{y} \cup B_{y-v}\right)+\mu\left(B_{x} \cup B_{x-v} \cup B_{y} \cup B_{y-v}\right)}\right] \mathrm{d} x \mathrm{~d} y \\
& \leq \int_{\mathbb{R}^{d} \times \mathbb{R}^{d}} \mathrm{e}^{-\mu\left(B_{x} \cup B_{x-v} \cup B_{y} \cup B_{y-v}\right)} \\
& \quad \times\left[1-\mathrm{e}^{-\mu\left(B_{x} \cap B_{y}\right)-\mu\left(B_{x} \cap B_{y-v}\right)-\mu\left(B_{x-v} \cap B_{y}\right)-\mu\left(B_{x-v} \cap B_{y-v}\right)}\right] \mathrm{d} x \mathrm{~d} y \\
& \leq \int_{\mathbb{R}^{d} \times \mathbb{R}^{d}} \mathrm{e}^{-\mu\left(B_{x} \cup B_{x-v} \cup B_{y} \cup B_{y-v}\right)}\left[1-\mathrm{e}^{-\mu\left(B_{x} \cap B_{y}\right)}+1-\mathrm{e}^{-\mu\left(B_{x} \cap B_{y-v}\right)}\right. \\
& \left.\quad+1-\mathrm{e}^{-\mu\left(B_{x-v} \cap B_{y}\right)}+1-\mathrm{e}^{-\mu\left(B_{x-v} \cap B_{y-v}\right)}\right] \mathrm{d} x \mathrm{~d} y,
\end{aligned}
$$

where the last inequality follows from the simple fact that

$$
1-\exp \left(-\sum_{i=1}^{k} a_{i}\right) \leq \sum_{i=1}^{k}\left(1-\mathrm{e}^{-a_{i}}\right) \quad \text { for } a_{i} \geq 0, i=1, \ldots, k
$$

Then $\operatorname{var}\left(\mu\left(C_{t} \cap\left(C_{t}+v\right)\right)\right) \leq \alpha_{1}+\alpha_{2}+\alpha_{3}+\alpha_{4}$, where

$$
\begin{aligned}
& \alpha_{1}:=\int_{\mathbb{R}^{d} \times \mathbb{R}^{d}} \mathrm{e}^{-\mu\left(B_{x} \cup B_{x-v} \cup B_{y} \cup B_{y-v}\right)}\left[1-\mathrm{e}^{-\mu\left(B_{x} \cap B_{y}\right)}\right] \mathrm{d} x \mathrm{~d} y, \\
& \alpha_{2}:=\int_{\mathbb{R}^{d} \times \mathbb{R}^{d}} \mathrm{e}^{-\mu\left(B_{x} \cup B_{x-v} \cup B_{y} \cup B_{y-v}\right)}\left[1-\mathrm{e}^{-\mu\left(B_{x} \cap B_{y-v}\right)}\right] \mathrm{d} x \mathrm{~d} y, \\
& \alpha_{3}:=\int_{\mathbb{R}^{d} \times \mathbb{R}^{d}} \mathrm{e}^{-\mu\left(B_{x} \cup B_{x-v} \cup B_{y} \cup B_{y-v}\right)}\left[1-\mathrm{e}^{-\mu\left(B_{x-v} \cap B_{y}\right)}\right] \mathrm{d} x \mathrm{~d} y, \\
& \alpha_{4}:=\int_{\mathbb{R}^{d} \times \mathbb{R}^{d}} \mathrm{e}^{-\mu\left(B_{x} \cup B_{x-v} \cup B_{y} \cup B_{y-v}\right)}\left[1-\mathrm{e}^{-\mu\left(B_{x-v} \cap B_{y-v}\right)}\right] \mathrm{d} x \mathrm{~d} y .
\end{aligned}
$$

We have

$$
\begin{aligned}
\alpha_{1} & \leq \int_{\mathbb{R}^{d} \times \mathbb{R}^{d}} \mathrm{e}^{-\mu\left(B_{x} \cup B_{y}\right)}\left[1-\mathrm{e}^{-\mu\left(B_{x} \cap B_{y}\right)}\right] \mathrm{d} x \mathrm{~d} y \\
& =\int_{\mathbb{R}^{d} \times \mathbb{R}^{d}}\left[\mathrm{e}^{-\mu\left(B_{x} \cup B_{y}\right)}-\mathrm{e}^{-\mu\left(B_{x}\right)-\mu\left(B_{y}\right)}\right] \mathrm{d} x \mathrm{~d} y \\
& =\operatorname{var}\left(\mu\left(C_{t}\right)\right),
\end{aligned}
$$

and similarly $\alpha_{i} \leq \operatorname{var}\left(\mu\left(C_{t}\right)\right), i=2,3,4$. This completes the proof. 
Remark 6. By Theorem 2 we have

$$
1 \leq \sup _{v \in \mathbb{R}^{d}} \frac{\operatorname{var}\left(\mu_{d}\left(C_{t, d} \cap\left(C_{t, d}+v\right)\right)\right)}{\operatorname{var}\left(\mu_{d}\left(C_{t, d}\right)\right)} \leq 4 .
$$

In view of Theorem 1, it seems natural to ask whether

$$
\operatorname{var}\left(\mu_{d}\left(C_{t, d} \cap\left(C_{t, d}+v\right)\right)\right) \leq \operatorname{var}\left(\mu_{d}\left(C_{t, d}\right)\right)
$$

for all $v \in \mathbb{R}^{d}$.

Remark 7. By a similar argument, it can be shown that, for every measurable set $S \subset \mathbb{R}^{d}$ and $v \in \mathbb{R}^{d}, \operatorname{var}\left(\mu_{d}\left(S \cap C_{t, d} \cap\left(C_{t, d}+v\right)\right)\right) \leq 4 \operatorname{var}\left(\mu_{d}\left(C_{t, d}\right)\right)$, and that, for $k=1,2, \ldots$ and $v_{1}, \ldots, v_{k} \in \mathbb{R}^{d}$,

$$
\operatorname{var}\left(\mu_{d}\left(C_{t, d} \cap\left(C_{t, d}+v_{1}\right) \cap \cdots \cap\left(C_{t, d}+v_{k}\right)\right)\right) \leq(k+1)^{2} \operatorname{var}\left(\mu_{d}\left(C_{t, d}\right)\right) .
$$

To see why a factor of $(k+1)^{2}$ appears in the latter inequality, we extend (7) and (8) to obtain, with $v_{0}:=\mathbf{0}, \mu:=\mu_{d}$, and $C_{t}:=C_{t, d}$,

$$
\begin{aligned}
\operatorname{var}\left(\mu\left(\bigcap_{i=0}^{k}\left(C_{t}+v_{i}\right)\right)\right)=\int_{\left(\mathbb{R}^{d}\right)^{2}} & {\left[\exp \left\{-\mu\left(\left(\bigcup_{i=0}^{k} B_{x-v_{i}}\right) \cup\left(\bigcup_{j=0}^{k} B_{y-v_{j}}\right)\right)\right\}\right.} \\
& \left.-\exp \left\{-\mu\left(\bigcup_{i=0}^{k} B_{x-v_{i}}\right)-\mu\left(\bigcup_{j=0}^{k} B_{y-v_{j}}\right)\right\}\right] \mathrm{d} x \mathrm{~d} y
\end{aligned}
$$

and

$$
\begin{aligned}
& \mu\left(\bigcup_{i=0}^{k} B_{x-v_{i}}\right)+\mu\left(\bigcup_{j=0}^{k} B_{y-v_{j}}\right)-\mu\left(\left(\bigcup_{i=0}^{k} B_{x-v_{i}}\right) \cup\left(\bigcup_{j=0}^{k} B_{y-v_{j}}\right)\right) \\
& \quad \leq \sum_{i=0}^{k} \sum_{j=0}^{k} \mu\left(B_{x-v_{i}} \cap B_{y-v_{j}}\right),
\end{aligned}
$$

which is a sum of $(k+1)^{2}$ terms. The desired inequality then follows along the lines of the proof of Theorem 2 .

Remark 8. By Theorem 2 we have $\mu_{d}\left(C_{t, d} \cap\left(C_{t, d}+r v_{d}\right)\right)$ converges in $L^{2}$ to $c(r, \lambda)$ for any sequence of $v_{d} \in \mathbb{R}^{d}$ satisfying $\left\|v_{d}\right\|_{d}=1, d=1,2, \ldots$, where $c(r, \lambda)$ is given in (2). Furthermore, we can use the argument in Remark 4 to strengthen the $L^{2}$ convergence to $L^{p}$ convergence for all $p>0$.

\section{Volume degeneracy of the typical cell of the $k$ th nearest-point Poisson-Voronoi tessellation}

Recall that $C_{t, d}^{(k)}$ as defined in (3) is the typical cell of the $k$ th nearest-point Poisson-Voronoi tessellation. Note that $\mathrm{E}\left[\mu_{d}\left(C_{t, d}^{(k)}\right)\right]=1 / \lambda$. We first prove the following main result of this section. 
Theorem 3. For $k=1,2, \ldots$ and $n=1,2, \ldots$, we have

$$
\lim _{d \rightarrow \infty} \mathrm{E}\left[\mu_{d}\left(C_{t, d}^{(k)}\right)\right]^{n}=\lambda^{-n} .
$$

Consequently, $\mu_{d}\left(C_{t, d}^{(k)}\right)$ converges in $L^{p}$ to $\lambda^{-1}$ as $d \rightarrow \infty$ for all $p>0$.

Proof. Without loss of generality, assume that $\lambda=1$. The case $k=1$ is contained in Theorem 10 of Newman et al. [11]. Their proof can be adapted to general $k$ as described below in detail. Letting $D_{k, d}:=\bigcup_{i=1}^{k} C_{t, d}^{(i)}$, since $\mathrm{E}\left[\mu_{d}\left(D_{k, d}\right)\right]=\sum_{i=1}^{k} \mathrm{E}\left[\mu_{d}\left(C_{t, d}^{(i)}\right)\right]=k$, Lemma 4, below, implies that $\operatorname{var}\left(\mu_{d}\left(D_{k, d}\right)\right) \rightarrow 0$ as $d \rightarrow \infty$, so that the distribution of $\mu_{d}\left(D_{k, d}\right)$ is asymptotically degenerate at $k$ as $d \rightarrow \infty$. By Lemma 5, below, we have, for all $k$ and $n$,

$$
\sup _{d} \mathrm{E}\left[\mu_{d}\left(D_{k, d}\right)\right]^{n}<\infty
$$

which together with the asymptotic degeneracy of $\mu_{d}\left(D_{k, d}\right)$ at $k$ implies that

$$
\lim _{d \rightarrow \infty} \mathrm{E}\left[\mu_{d}\left(D_{k, d}\right)\right]^{n}=k^{n}
$$

Since $\mu_{d}\left(C_{t, d}^{(k)}\right)=\mu_{d}\left(D_{k, d}\right)-\mu_{d}\left(D_{k-1, d}\right)$, where $D_{0, d}:=\varnothing$, the theorem follows.

Lemma 1. Let $D_{k, d}:=\bigcup_{i=1}^{k} C_{t, d}^{(i)}$. Then, for $x, y \in \mathbb{R}^{d}$,

$$
\mathrm{E}\left[\mathbf{1}_{D_{k, d}}(x) \mathbf{1}_{D_{k, d}}(y)\right] \geq \mathrm{E}\left[\mathbf{1}_{D_{k, d}}(x)\right] \mathrm{E}\left[\mathbf{1}_{D_{k, d}}(y)\right] .
$$

Proof. Let $N_{1}:=\#\left(\Pi \cap\left(B_{x} \backslash B_{y}\right)\right)$ (the number of $\Pi$-points in $\left.B_{x} \backslash B_{y}\right), N_{2}:=\#(\Pi \cap$ $\left.\left(B_{y} \backslash B_{x}\right)\right)$, and $N_{3}:=\#\left(\Pi \cap B_{x} \cap B_{y}\right)$. Then $N_{1}, N_{2}$, and $N_{3}$ are independent Poisson random variables, and $\left\{x \in D_{k, d}\right\}=\left\{N_{1}+N_{3} \leq k-1\right\}$ and $\left\{y \in D_{k, d}\right\}=\left\{N_{2}+N_{3} \leq k-1\right\}$. It can be readily shown that the conditional distribution of $N_{3}$ given that $N_{1}+N_{3} \leq k-1$ is stochastically smaller than the (unconditional) distribution of $N_{3}$, which implies that the conditional distribution of $N_{2}+N_{3}$ given that $N_{1}+N_{3} \leq k-1$ is stochastically smaller than the (unconditional) distribution of $N_{2}+N_{3}$. It follows that

$$
\mathrm{P}\left(N_{2}+N_{3} \leq k-1 \mid N_{1}+N_{3} \leq k-1\right) \geq \mathrm{P}\left(N_{2}+N_{3} \leq k-1\right),
$$

which is equivalent to the desired inequality, completing the proof.

For the lemmas below, assume that $\lambda=1$. For given $-1 \leq w \leq 1, \rho_{1}>0$, and $\rho_{2}>0$, let $x, y \in \mathbb{R}^{d}$ be any points satisfying

$$
\rho_{1}=\mu_{d}\left(B_{x}\right)=v_{d}\|x\|_{d}^{d}, \quad \rho_{2}=\mu_{d}\left(B_{y}\right)=v_{d}\|y\|_{d}^{d}, \quad w=\frac{\langle x, y\rangle_{d}}{\|x\|_{d}\|y\|_{d}},
$$

where $v_{d}=\pi^{d / 2} / \Gamma(d / 2+1)$ is the $d$-volume of the unit ball in $\mathbb{R}^{d}$. Let

$$
P_{k, d}\left(w, \rho_{1}, \rho_{2}\right):=\mathrm{P}\left\{\#\left(\Pi \cap B_{x}\right) \leq k-1, \#\left(\Pi \cap B_{y}\right) \leq k-1\right\},
$$

which depends on $x$ and $y$ only through $w, \rho_{1}$, and $\rho_{2}$. Note that

$$
P_{k, d}\left(w, \rho_{1}, \rho_{2}\right)=\mathrm{E}\left[\mathbf{1}_{D_{k, d}}(x) \mathbf{1}_{D_{k, d}}(y)\right]
$$

for $x$ and $y$ satisfying (9). Let $W_{d}:=\langle X, Y\rangle_{d}$, where $X$ and $Y$ are independent and uniformly distributed on the unit sphere $S^{d-1}$ in $\mathbb{R}^{d}$. It can be shown (cf. [11, p. 742]) that the density function of $W_{d}, f_{d}(w)$, is proportional to $\left(1-w^{2}\right)^{(d-3) / 2},-1 \leq w \leq 1$. 
Lemma 2. Assume that $\lambda=1$. We have

$$
\mathrm{E}\left[\mu_{d}\left(D_{k, d}\right)\right]^{2}=\int_{0}^{\infty} \int_{0}^{\infty} \int_{-1}^{1} P_{k, d}\left(w, \rho_{1}, \rho_{2}\right) f_{d}(w) \mathrm{d} w \mathrm{~d} \rho_{1} \mathrm{~d} \rho_{2} .
$$

Proof. For notational simplicity, write $\mu=\mu_{d}, D=D_{k, d}$, and $\langle\cdot, \cdot\rangle=\langle\cdot, \cdot\rangle_{d}$. We have

$$
\begin{aligned}
\mathrm{E}[\mu(D)]^{2} & =\int_{\left(\mathbb{R}^{d}\right)^{2}} \mathrm{E}\left[\mathbf{1}_{D}(x) \mathbf{1}_{D}(y)\right] \mathrm{d} x \mathrm{~d} y \\
& =\int_{0}^{\infty} \int_{0}^{\infty} M\left(r_{1}, r_{2}\right) \sigma_{d} r_{1}^{d-1} \mathrm{~d} r_{1} \sigma_{d} r_{2}^{d-1} \mathrm{~d} r_{2},
\end{aligned}
$$

where $\sigma_{d}=2 \pi^{d / 2} / \Gamma(d / 2)$ is the $(d-1)$-volume (surface area) of the unit sphere $S^{d-1}$, and $M\left(r_{1}, r_{2}\right)$ is the average value of $\mathrm{E}\left[\mathbf{1}_{D}(x) \mathbf{1}_{D}(y)\right]$ over $x \in r_{1} S^{d-1}$ and $y \in r_{2} S^{d-1}$ (spheres of radii $r_{1}$ and $\left.r_{2}\right)$. To find $M\left(r_{1}, r_{2}\right)$, note that $\mathrm{E}\left[\mathbf{1}_{D}(x) \mathbf{1}_{D}(y)\right]$ depends on $x \in r_{1} S^{d-1}$ and $y \in r_{2} S^{d-1}$ only through $r_{1}, r_{2}$, and $w=\langle x, y\rangle / r_{1} r_{2}$. For given $w, r_{1}$, and $r_{2}$, let

$$
L\left(w, r_{1}, r_{2}\right):=\mathrm{E}\left[\mathbf{1}_{D}(x) \mathbf{1}_{D}(y)\right]
$$

for any $x \in r_{1} S^{d-1}$ and $y \in r_{2} S^{d-1}$ satisfying $w=\langle x, y\rangle / r_{1} r_{2}$. Then the average value $M\left(r_{1}, r_{2}\right)$ of $\mathrm{E}\left[\mathbf{1}_{D}(x) \mathbf{1}_{D}(y)\right]$ over $x \in r_{1} S^{d-1}$ and $y \in r_{2} S^{d-1}$ equals

$$
\int_{-1}^{1} L\left(w, r_{1}, r_{2}\right) f_{d}(w) \mathrm{d} w
$$

since $f_{d}(w)$ is the density of $w=\langle x, y\rangle / r_{1} r_{2}$ with $x / r_{1}$ and $y / r_{2}$ interpreted as independent random variables uniformly distributed on $S^{d-1}$. Thus, by (11),

$$
\mathrm{E}[\mu(D)]^{2}=\int_{0}^{\infty} \int_{0}^{\infty} \int_{-1}^{1} L\left(w, r_{1}, r_{2}\right) f_{d}(w) \mathrm{d} w \sigma_{d} r_{1}^{d-1} \mathrm{~d} r_{1} \sigma_{d} r_{2}^{d-1} \mathrm{~d} r_{2} .
$$

Letting $\rho_{i}:=\sigma_{d} r_{i}^{d} / d\left(=v_{d} r_{i}^{d}\right), i=1,2$, note that $x$ and $y$ satisfy $x \in r_{1} S^{d-1}, y \in r_{2} S^{d-1}$, and $w=\langle x, y\rangle / r_{1} r_{2}$ if and only if $x$ and $y$ satisfy (9). So, by (10) and (12), $L\left(w, r_{1}, r_{2}\right)=$ $P_{k, d}\left(w, \rho_{1}, \rho_{2}\right)$, which together with (13) completes the proof.

Lemma 3. For $-1 \leq w \leq 1, \rho_{1}>0$, and $\rho_{2}>0$, let $x$ and $y$ be any points satisfying (9). Then

$$
\mu_{d}\left(B_{x} \cap B_{y}\right) \leq\left(\frac{1+w}{2}\right)^{d / 2} \max \left\{\rho_{1}, \rho_{2}\right\} .
$$

Proof. Let $\rho:=\max \left\{\rho_{1}, \rho_{2}\right\}, x^{\prime}:=\left(\rho / \rho_{1}\right)^{1 / d} x, y^{\prime}:=\left(\rho / \rho_{2}\right)^{1 / d} y$, so that $\mu_{d}\left(B_{x^{\prime}}\right)=$ $\mu_{d}\left(B_{y^{\prime}}\right)=\rho$ and $\left\langle x^{\prime}, y^{\prime}\right\rangle_{d} /\left\|x^{\prime}\right\|_{d}\left\|y^{\prime}\right\|_{d}=w$, and $B_{x} \cap B_{y} \subset B_{x^{\prime}} \cap B_{y^{\prime}}$. Since the angle $\theta$ between the line segments $\overline{x^{\prime} \mathbf{0}}$ and $\overline{y^{\prime} \mathbf{0}}$ satisfies $\cos \theta=w(0 \leq \theta \leq \pi)$, the intersection of $B_{x^{\prime}} \cap B_{y^{\prime}}$ with the $(d-1)$-dimensional hyperplane passing through $\left(x^{\prime}+y^{\prime}\right) / 2$ and perpendicular to $\overline{x^{\prime} y^{\prime}}$, is a $(d-1)$-dimensional ball centered at $\left(x^{\prime}+y^{\prime}\right) / 2$ with radius $\left\|\left(x^{\prime}+y^{\prime}\right) / 2\right\|_{d}=$ $\left\|x^{\prime}\right\|_{d} \cos (\theta / 2)=\left(\rho / \nu_{d}\right)^{1 / d} \cos (\theta / 2)$. It is readily seen that $B_{x^{\prime}} \cap B_{y^{\prime}}$ is contained in the ( $d$-dimensional) ball centered at $\left(x^{\prime}+y^{\prime}\right) / 2$ with radius $\left(\rho / v_{d}\right)^{1 / d} \cos (\theta / 2)$. It follows that

$$
\mu_{d}\left(B_{x} \cap B_{y}\right) \leq \mu_{d}\left(B_{x^{\prime}} \cap B_{y^{\prime}}\right) \leq \rho \cos ^{d}\left(\frac{\theta}{2}\right)=\rho\left(\frac{1+w}{2}\right)^{d / 2},
$$

completing the proof. 
Lemma 4. Assume that $\lambda=1$. As $d \rightarrow \infty, \mathrm{E}\left[\mu_{d}\left(D_{k, d}\right)\right]^{2} \rightarrow k^{2}$.

Proof. For $\rho_{1}>0$ and $\rho_{2}>0$, let

$$
G_{d}\left(\rho_{1}, \rho_{2}\right):=\int_{-1}^{1} P_{k, d}\left(w, \rho_{1}, \rho_{2}\right) f_{d}(w) \mathrm{d} w
$$

so that, by Lemma 2,

$$
\mathrm{E}\left[\mu_{d}\left(D_{k, d}\right)\right]^{2}=\int_{0}^{\infty} \int_{0}^{\infty} G_{d}\left(\rho_{1}, \rho_{2}\right) \mathrm{d} \rho_{1} \mathrm{~d} \rho_{2} .
$$

By (10), for $x$ and $y$ satisfying (9),

$$
P_{k, d}\left(w, \rho_{1}, \rho_{2}\right)=\mathrm{E}\left[\mathbf{1}_{D_{k, d}}(x) \mathbf{1}_{D_{k, d}}(y)\right] \leq \mathrm{E}\left[\mathbf{1}_{D_{k, d}}(z)\right]=\mathrm{P}\left\{\#\left(\Pi \cap B_{z}\right) \leq k-1\right\},
$$

where $z:=x \mathbf{1}_{\rho_{1} \geq \rho_{2}}+y \mathbf{1}_{\rho_{1}<\rho_{2}}$. Since $\mu_{d}\left(B_{z}\right)=\max \left\{\rho_{1}, \rho_{2}\right\}$,

$$
P_{k, d}\left(w, \rho_{1}, \rho_{2}\right) \leq \sum_{i=0}^{k-1} \frac{\left(\max \left\{\rho_{1}, \rho_{2}\right\}\right)^{i}}{i !} \mathrm{e}^{-\max \left\{\rho_{1}, \rho_{2}\right\}} \leq c_{k} \mathrm{e}^{-\max \left\{\rho_{1}, \rho_{2}\right\} / 2},
$$

where

$$
c_{k}:=\sup _{u>0} \sum_{i=0}^{k-1} \frac{u^{i}}{i !} \mathrm{e}^{-u / 2}<\infty .
$$

It follows by (14) that $G_{d}\left(\rho_{1}, \rho_{2}\right) \leq c_{k} \mathrm{e}^{-\max \left\{\rho_{1}, \rho_{2}\right\} / 2}$. If we can show that

$$
\lim _{d \rightarrow \infty} G_{d}\left(\rho_{1}, \rho_{2}\right)=\left(\sum_{i=0}^{k-1} \frac{\rho_{1}^{i}}{i !} \mathrm{e}^{-\rho_{1}}\right)\left(\sum_{i=0}^{k-1} \frac{\rho_{2}^{i}}{i !} \mathrm{e}^{-\rho_{2}}\right) \text { for } \rho_{1}>0 \text { and } \rho_{2}>0,
$$

then it follows from the dominated convergence theorem and (15) that

$$
\lim _{d \rightarrow \infty} \mathrm{E}\left[\mu_{d}\left(D_{k, d}\right)\right]^{2}=\int_{0}^{\infty} \int_{0}^{\infty}\left(\sum_{i=0}^{k-1} \frac{\rho_{1}^{i}}{i !} \mathrm{e}^{-\rho_{1}}\right)\left(\sum_{i=0}^{k-1} \frac{\rho_{2}^{i}}{i !} \mathrm{e}^{-\rho_{2}}\right) \mathrm{d} \rho_{1} \mathrm{~d} \rho_{2}=k^{2} .
$$

It remains to establish (18). For fixed $\rho_{1}$ and $\rho_{2}$, we have, by (16),

$$
\int_{1 / 2}^{1} P_{k, d}\left(w, \rho_{1}, \rho_{2}\right) f_{d}(w) \mathrm{d} w \leq c_{k} \mathrm{e}^{-\max \left\{\rho_{1}, \rho_{2}\right\} / 2} \int_{1 / 2}^{1} f_{d}(w) \mathrm{d} w \rightarrow 0 \quad \text { as } d \rightarrow \infty .
$$

For $-1 \leq w \leq \frac{1}{2}$, we have, by (10) with $x$ and $y$ satisfying (9),

$$
\begin{aligned}
& P_{k, d}\left(w, \rho_{1}, \rho_{2}\right) \\
&= \mathrm{P}\left\{\#\left(\Pi \cap B_{x}\right) \leq k-1, \#\left(\Pi \cap B_{y}\right) \leq k-1\right\} \\
& \leq \mathrm{P}\left\{\#\left(\Pi \cap B_{x} \cap B_{y}\right)>0\right\} \\
&+\mathrm{P}\left\{\#\left(\Pi \cap\left(B_{x} \backslash B_{y}\right)\right) \leq k-1, \#\left(\Pi \cap\left(B_{y} \backslash B_{x}\right)\right) \leq k-1\right\} \\
&= 1-\mathrm{e}^{-u(w)}+\left(\sum_{i=0}^{k-1} \frac{\left(\rho_{1}-u(w)\right)^{i}}{i !} \mathrm{e}^{-\rho_{1}+u(w)}\right)\left(\sum_{i=0}^{k-1} \frac{\left(\rho_{2}-u(w)\right)^{i}}{i !} \mathrm{e}^{-\rho_{2}+u(w)}\right) \\
& \rightarrow\left(\sum_{i=0}^{k-1} \frac{\rho_{1}^{i}}{i !} \mathrm{e}^{-\rho_{1}}\right)\left(\sum_{i=0}^{k-1} \frac{\rho_{2}^{i}}{i !} \mathrm{e}^{-\rho_{2}}\right) \text { as } d \rightarrow \infty,
\end{aligned}
$$


uniformly in $-1 \leq w \leq \frac{1}{2}$, where $u(w):=\mu_{d}\left(B_{x} \cap B_{y}\right) \leq[(1+w) / 2]^{d / 2} \max \left\{\rho_{1}, \rho_{2}\right\}$ by Lemma 3. On the other hand, by Lemma 1 we have, for $-1 \leq w \leq 1$ and $x$ and $y$ satisfying (9),

$$
\begin{aligned}
P_{k, d}\left(w, \rho_{1}, \rho_{2}\right) & =\mathrm{E}\left[\mathbf{1}_{D_{k, d}}(x) \mathbf{1}_{D_{k, d}}(y)\right] \\
& \geq \mathrm{E}\left[\mathbf{1}_{D_{k, d}}(x)\right] \mathrm{E}\left[\mathbf{1}_{D_{k, d}}(y)\right] \\
& =\left(\sum_{i=0}^{k-1} \frac{\rho_{1}^{i}}{i !} \mathrm{e}^{-\rho_{1}}\right)\left(\sum_{i=0}^{k-1} \frac{\rho_{2}^{i}}{i !} \mathrm{e}^{-\rho_{2}}\right),
\end{aligned}
$$

which together with (19) and (20) implies (18). The proof is complete.

Lemma 5. Assume that $\lambda=1$. We have

$$
\mathrm{E}\left[\mu_{d}\left(D_{k, d}\right)\right]^{n} \leq c_{k} 2^{n} n !, \quad n=1,2, \ldots,
$$

where $c_{k}$ is as defined in (17).

Proof. Write $\mu=\mu_{d},\|\cdot\|=\|\cdot\|_{d}$, and $D=D_{k, d}$ for notational simplicity. By Fubini's theorem,

$$
\begin{aligned}
\mathrm{E}[\mu(D)]^{n} & =\int_{\left(\mathbb{R}^{d}\right)^{n}} \mathrm{E}\left[\mathbf{1}_{D}\left(x_{1}\right) \cdots \mathbf{1}_{D}\left(x_{n}\right)\right] \mathrm{d} x_{1} \cdots \mathrm{d} x_{n} \\
& \leq \int_{\left(\mathbb{R}^{d}\right)^{n}} \mathrm{E}\left[\mathbf{1}_{D}\left(x_{I(n)}\right)\right] \mathrm{d} x_{1} \cdots \mathrm{d} x_{n},
\end{aligned}
$$

where $I(n)=I\left(n, x_{1}, \ldots, x_{n}\right):=\min \left\{1 \leq i \leq n:\left\|x_{i}\right\| \geq\left\|x_{j}\right\|\right.$ for all $\left.1 \leq j \leq n\right\}$. We have

$$
\mathrm{E}\left[\mathbf{1}_{D}\left(x_{I(n)}\right)\right]=\mathrm{P}\left\{\#\left(\Pi \cap B_{x_{I(n)}}\right) \leq k-1\right\}=\sum_{i=0}^{k-1} \frac{u^{i}}{i !} \mathrm{e}^{-u} \leq c_{k} \mathrm{e}^{-u / 2},
$$

where $u:=\mu\left(B_{x_{I(n)}}\right)=\max \left\{\mu\left(B_{x_{i}}\right): i=1, \ldots, n\right\}$. It follows from (21) and (22) that

$$
\mathrm{E}[\mu(D)]^{n} \leq c_{k} \int_{\left(\mathbb{R}^{d}\right)^{n}} \exp \left(-\frac{1}{2} \max _{1 \leq i \leq n} \mu\left(B_{x_{i}}\right)\right) \mathrm{d} x_{1} \cdots \mathrm{d} x_{n}=c_{k} n !\left(\frac{1}{2}\right)^{-n},
$$

where the equality is derived in the proof of Lemma 3.1 in [1, pp. 922, 923]. The proof is complete.

Remark 9. Similarly to Theorem 1, it is easily shown from Lemma 1 that the covariance of $\mu_{d}\left(D_{k, d} \cap S_{1}\right)$ and $\mu_{d}\left(D_{k, d} \cap S_{2}\right)$ is nonnegative for any measurable subsets $S_{1}$ and $S_{2}$ of $\mathbb{R}^{d}$. (Note that the nonnegative covariance property does not hold for $C_{t, d}^{(k)}$ with $k \geq 2$.) It then follows that

$$
\operatorname{var}\left(\mu_{d}\left(D_{k, d} \cap S_{1}\right)\right) \leq \operatorname{var}\left(\mu_{d}\left(D_{k, d} \cap S_{2}\right)\right) \leq \operatorname{var}\left(\mu_{d}\left(D_{k, d}\right)\right),
$$

if $S_{1} \subset S_{2} \subset \mathbb{R}^{d}$. So, by Theorem 3 for any measurable sets $A_{d} \subset \mathbb{R}^{d}, \mu_{d}\left(D_{k, d} \cap A_{d}\right)-$ $\mathrm{E}\left[\mu_{d}\left(D_{k, d} \cap A_{d}\right)\right]$ converges in $L^{2}$ to 0 as $d \rightarrow \infty$. Since $\mu_{d}\left(C_{t, d}^{(k)} \cap A_{d}\right)=\mu_{d}\left(D_{k, d} \cap A_{d}\right)-$ $\mu_{d}\left(D_{k-1, d} \cap A_{d}\right)$, we have $\mu_{d}\left(C_{t, d}^{(k)} \cap A_{d}\right)-\mathrm{E}\left[\mu_{d}\left(C_{t, d}^{(k)} \cap A_{d}\right)\right]$ converges in $L^{2}$ to 0 as $d \rightarrow \infty$. By the argument in Remark 4 , the $L^{2}$ convergence can be strengthened to $L^{p}$ convergence for all $p>0$. 
Remark 10. Along the lines of the proof of Lemma 4 with more refined calculations, it can be shown that $\operatorname{var}\left(\mu_{d}\left(D_{k, d}\right)\right)$ decays to 0 at a geometric rate as $d \rightarrow \infty$, which in turn implies that $\operatorname{var}\left(\mu_{d}\left(C_{t, d}^{(k)}\right)\right)$ decays to 0 at a geometric rate as $d \rightarrow \infty$. Define, for $k=1,2, \ldots$,

$$
\rho_{k}:=\inf \left\{\rho>0: \sup _{d} \frac{\operatorname{var}\left(\mu_{d}\left(C_{t, d}^{(k)}\right)\right)}{\rho^{d}}<\infty\right\} .
$$

While Alishahi and Sharifitabar [1] obtained $\rho_{1}=4 / 3 \sqrt{3}$, it is of theoretical interest to find the exact value of $\rho_{k}$ for $k \geq 2$ as well as to study the qualitative behavior of the $\rho_{k}$. In particular, it seems of interest to know whether the $\rho_{k}$ are monotone in $k$.

Remark 11. Newman et al. [11, Theorem 10] considered a two-ensemble $d$-dimensional Poisson point process with $\Pi_{1}$-points of intensity $\lambda_{1}$ and $\Pi_{2}$-points of intensity $\lambda_{2}$. Let $Q_{\Pi_{1}}$ denote a typical $\Pi_{1}$-point, and let $N_{k, d}, k=1,2, \ldots$, be the number of $\Pi_{2}$-points that have $Q_{\Pi_{1}}$ as their $k$ th nearest $\Pi_{1}$-neighbor. Newman et al. proved that, as $d \rightarrow \infty, N_{1, d}$ is asymptotically Poisson with mean $\lambda_{2} / \lambda_{1}$. Denote by $C_{t, d, \Pi_{1}}^{(k)}$ the (typical) $k$ th nearest-point Voronoi cell centered at $Q_{\Pi_{1}}$ generated by the $\Pi_{1}$-points. Then $N_{k, d}$ is the number of $\Pi_{2}$-points in $C_{t, d, \Pi_{1}}^{(k)}$. Since, as $d \rightarrow \infty, \mu_{d}\left(C_{t, d, \Pi_{1}}^{(k)}\right)$ is asymptotically degenerate at $1 / \lambda_{1}, k=1,2, \ldots$, and since, conditional on $\mu_{d}\left(C_{t, d, \Pi_{1}}^{(k)}\right), k=1,2, \ldots$, the $N_{k, d}$ are independent Poisson random variables with means $\lambda_{2} \mu_{d}\left(C_{t, d, \Pi_{1}}^{(k)}\right)$, it follows that, as $d \rightarrow \infty$, the $N_{k, d}, k=1,2, \ldots$, are asymptotically independent and identically distributed Poisson with mean $\lambda_{2} / \lambda_{1}$.

\section{Acknowledgements}

The author is grateful to the anonymous referee for useful comments which led to an improved presentation. The author also gratefully acknowledges support from the National Science Council of Taiwan under grant NSC 096-2628-M-001-023.

\section{References}

[1] Alishahi, K. and Sharifitabar, M. (2008). Volume degeneracy of the typical cell and the chord length distribution for Poisson-Voronoi tessellations in high dimensions. Adv. Appl. Prob. 40, 919-938.

[2] Averkov, G. and Bianchi, G. (2007). Retrieving convex bodies from restricted covariogram functions. Adv. Appl. Prob. 39, 613-629.

[3] Bianchi, G. (2005). Matheron's conjecture for the covariogram problem. J. London Math. Soc. 71, $203-220$.

[4] Gilbert, E. N. (1962). Random subdivisions of space into crystals. Ann. Math. Statist. 33, 958-972.

[5] Hinde, A. L. And Miles, R. E. (1980). Monte Carlo estimates of the distributions of the random polygons of the Voronoi tessellation with respect to a Poisson process. J. Statist. Comput. Simul. 10, 205-223.

[6] Jensen, E. B. V. (1998). Local Stereology (Adv. Ser. Statist. Sci. Appl. Prob. 5). World Scientific, River Edge, NJ.

[7] LantuéJoul, C. (2002). Geostatistical Simulation: Models and Algorithms. Springer, Berlin.

[8] Matheron, G. (1975). Random Sets and Integral Geometry. John Wiley, New York.

[9] MøLler, J. (1994). Lectures on Random Voronoi Tessellations (Lecture Notes Statist. 87). Springer, New York.

[10] Newman, C. M. And RinotT, Y. (1985). Nearest neighbors and Voronoi volumes in high-dimensional point processes with various distance functions. Adv. Appl. Prob. 17, 794-809.

[11] Newman, C. M., Rinott, Y. and Tversky, A. (1983). Nearest neighbors and Voronoi regions in certain point processes. Adv. Appl. Prob. 15, 726-751.

[12] Okabe, A., Boots, B., Sugihara, K. And Chiu, S. N. (2000). Spatial Tessellations: Concepts and Applications of Voronoi Diagrams, 2nd edn. John Wiley, Chichester.

[13] YaO, Y.-C. And Simons, G. (1996). A large-dimensional independent and identically distributed property for nearest neighbor counts in Poisson processes. Ann. Appl. Prob. 6, 561-571. 